\title{
Characterization of Photoconductive Diamond Detectors - Candidate Vacuum Ultraviolet Radiation and Extreme Ultraviolet Radiation Light Source Detectors for Lithography -
}

\author{
Hideaki Ishihara*, Shoshiro Sugio, Toshiya Kanno, \\ Mikihiko Matsuoka and Kazushi Hayashi ${ }^{1}$ \\ Corporate Advanced Technology, Iwasaki Electric Co., Ltd. \\ ${ }^{1}$ Electronics Research Laboratory, Kobe Steel, Ltd.
}

(Received February 4, 2010; accepted April 27, 2010)

Key words: photoconductive diamond detector, vacuum ultraviolet (VUV) radiation, extreme ultraviolet (EUV) radiation, VUV/EUV radiation light source detectors for lithography

The characteristics of an ultraviolet (UV) and vacuum ultraviolet (VUV) radiation photodetector with a photoconductive highly oriented diamond (HOD) film were evaluated using continuous light sources (a low-pressure mercury lamp and a xenon excimer lamp) that mainly emit light in the UV range. It was confirmed that the change in the spectral responsivity of the photodetector is extremely limited upon continuous UV radiation. In this report, the results of the evaluation of the characteristics of the UV photodetector with a photoconductive diamond film (hereafter, diamond UV photodetector) using continuous light sources are reported. We also found that the diamond UV photodetector has characteristics enabling its use as a photodetector for ArF excimer lasers and extreme ultraviolet (EUV) light sources, as a result of the evaluation using a light source employed in lithography. The results are also described. The evaluation of the diamond UV photodetector using an ArF excimer laser revealed that it can detect a laser with a pulse width of $5 \mathrm{~ns}$. Also, good linearity between the pulse energy and the output was observed for pulse energies of $1-100 \mu \mathrm{J} / \mathrm{cm}^{2}$. By the measurement of the spectral responsivity to synchrotron radiation, the diamond UV photodetector was found to have high spectral responsivity in the wavelength range of $10-33 \mathrm{~nm}$. According to the results given above, the diamond UV photodetector has sufficient time responsiveness (follow-up performance) to enable the measurement of ArF excimer lasers and sufficient spectral responsivity to detect light in the EUV region, suggesting its use as a photodetector of light sources used in lithography.

*Corresponding author: e-mail: ishihara-hideaki@eye.co.jp 


\section{Introduction}

Vacuum ultraviolet (VUV) radiation light sources, represented by an ArF excimer laser (wavelength: $193 \mathrm{~nm}$ ), a low-pressure mercury lamp (wavelength: $185 \mathrm{~nm}$ ), and a xenon excimer lamp (wavelength: $172 \mathrm{~nm}$ ), have recently been used in various industrial applications, such as for the sterilization of water and air, the dry cleaning of semiconductors and flat panel displays, and as light sources for exposure apparatus. In particular, the research and development of an EUV light source (wavelength: $13.5 \mathrm{~nm}$ ) has been carried out to investigate its potential as a next-generation light source for exposure apparatus and as a replacement of conventional ArF excimer lasers, to improve the resolution of exposure apparatus. ${ }^{(1)}$ The important characteristics required for photodetectors used to measure VUV include the linearity and stability of spectral responsivity and the detection of the laser only in the target wavelength region while remaining unaffected by light of other wavelengths. Also, responsiveness within the timescale of one laser pulse is necessary for the measurement of a pulse light source such as an excimer laser.

In this report, we report the characteristics of the diamond UV photodetector with a highly oriented diamond film examined using continuous light sources (a low-pressure mercury lamp and a xenon excimer lamp) that emit light in the UV range. Furthermore, the feasibility of the diamond UV photodetector as a photodetector for light sources for exposure apparatus is examined using an ArF excimer laser (which is currently generally used as a light source for exposure apparatus) and an EUV light source (which is expected to be used as a next-generation light source for exposure apparatus).

\section{Experimental}

Diamond is a material that possesses excellent chemical inertness to and durability against intense UV radiation. In addition, because the band gap energy $(5.47 \mathrm{eV})$ of diamond corresponds to a wavelength of approximately $227 \mathrm{~nm}$, it is sensitive to UV radiation only. Therefore, the diamond UV photodetector, different from existing UV detectors, is capable of directly detecting UV without the aid of an optical filter (less durable UV radiation) that blocks light (wavelength: $227 \mathrm{~nm}$ or longer) other than UV.(2-4)

The diamond UV photodetectors used HOD films made by Kobe Steel, Ltd..$^{(5-7)}$ The HOD films were produced on silicon (001) substrates by microwave plasma chemical vapor deposition (CVD) with bias-enhanced nucleation (BEN) treatment. The HOD films consisted of azimuthally oriented (001) facets with typical grains from 1 to $3 \mu \mathrm{m}$ sizes and a thickness of $10 \mu \mathrm{m}$. A pair of interdigitated Pt electrodes was fabricated on the HOD film surface. The thickness of the Pt electrodes was approximately $0.2 \mu \mathrm{m}$, the distance between the electrodes was $10 \mu \mathrm{m}$, and the electrode width was $5 \mu \mathrm{m}$. The patterned area of the interdigitated electrodes was $2 \times 2 \mathrm{~mm}^{2}$, and the total size of the sensor chip was $3 \times 3 \mathrm{~mm}^{2}$.

The sensor chips were finally mounted on a hermetic seal (standard TO5type package) and wire-bonded to lead pins using gold wires. A sapphire window having a cutoff wavelength of approximately $140 \mathrm{~nm}$ was used as the seal cap, and the inside was purged with argon gas. Figure 1 shows the packaged diamond UV photodetectors. 


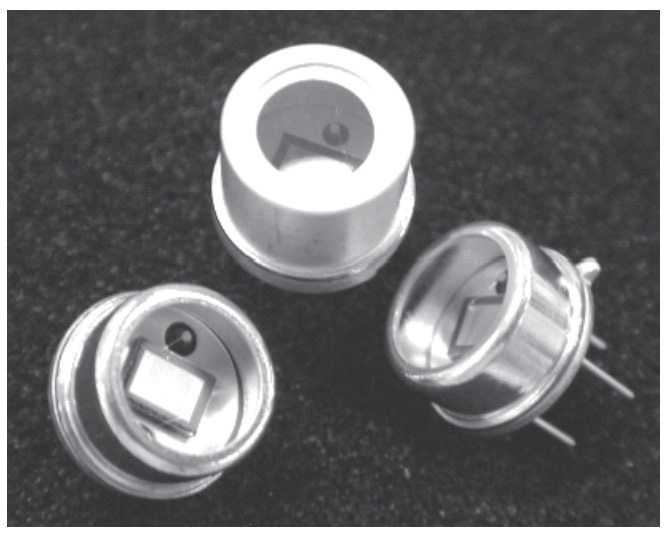

Fig. 1. Appearance of diamond UV photodetectors.

\section{Results and Discussion}

\subsection{ArF excimer laser (wavelength: $193 \mathrm{~nm}$ )}

The time responsiveness and linearity of the diamond UV photodetector upon the irradiation of an ArF excimer laser (PSX-100, MPB Communications Inc.) were examined. A bias voltage of $5 \mathrm{~V}$ was applied to the photodetector during measurement.

Figure 2 shows the relative photocurrent of the diamond UV photodetector upon the irradiation of a laser with a pulse width of $5 \mathrm{~ns}$. A peak in the relative photocurrent is observed at $5 \mathrm{~ns}$. This confirms that the diamond UV photodetector has a time responsiveness equivalent to that of the conventional sensors (silicon photodetectors).

Figure 3 shows the relationship between the pulse energy of the laser irradiated and the output from the diamond UV photodetector. In the region where the pulse energy of the irradiated laser is small, a linearity between the pulse energy and the output was observed, whereas in the region where the pulse energy is large and the photodetector output is equivalent to that of the bias voltage, the photodetector output tends to saturate, i.e., a linearity between the pulse energy and the output of the diamond UV photodetector was observed for the ArF excimer laser with a pulse energy range of 1-100 $\mu \mathrm{J} / \mathrm{cm}^{2}$.

\subsection{Synchrotron radiation (wavelength: 10-33 $\mathrm{nm}$ )}

The spectral responsivity measurements in the EUV spectral range from 10 to 33 $\mathrm{nm}$ were performed using synchrotron radiation from the electron storage ring, TERAS at the National Institute of Advanced Industrial Science and Technology (AIST). The National Metrology Institute of Japan (NMIJ) provides the calibration service of spectral responsivity in the wavelength range of 10 to $33 \mathrm{~nm} \cdot{ }^{(8-11)}$ We asked the NMIJ to calibrate the spectral responsivity of the diamond UV photodetector for light in the EUV range. For measurement, a large-area highly oriented diamond film $\left(7.5 \times 7.5 \mathrm{~mm}^{2}\right)$ was used as the diamond UV photodetector, and in the packaging, no sapphire cap was used to 


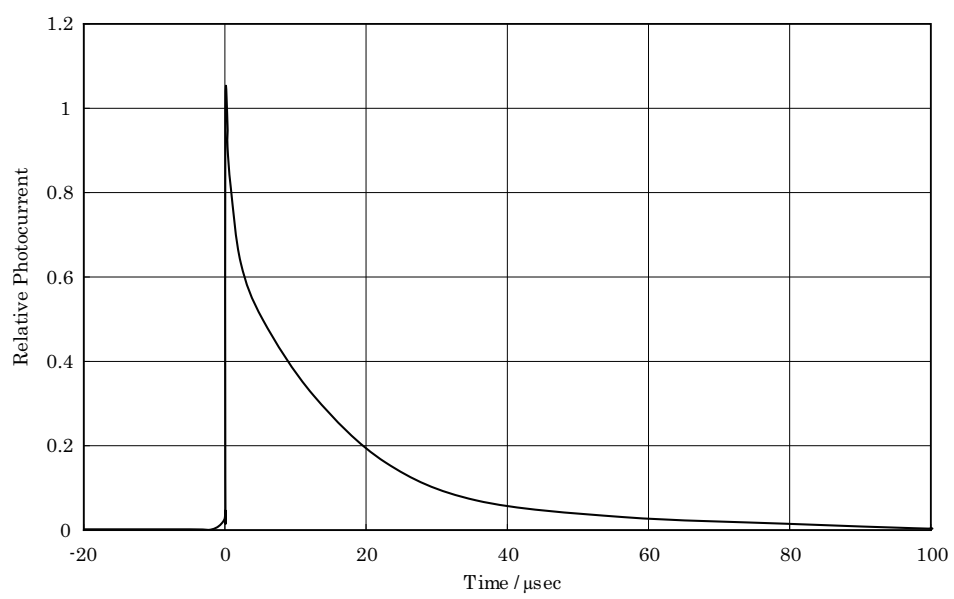

Fig. 2. Time responsiveness upon irradiation of ArF excimer laser.

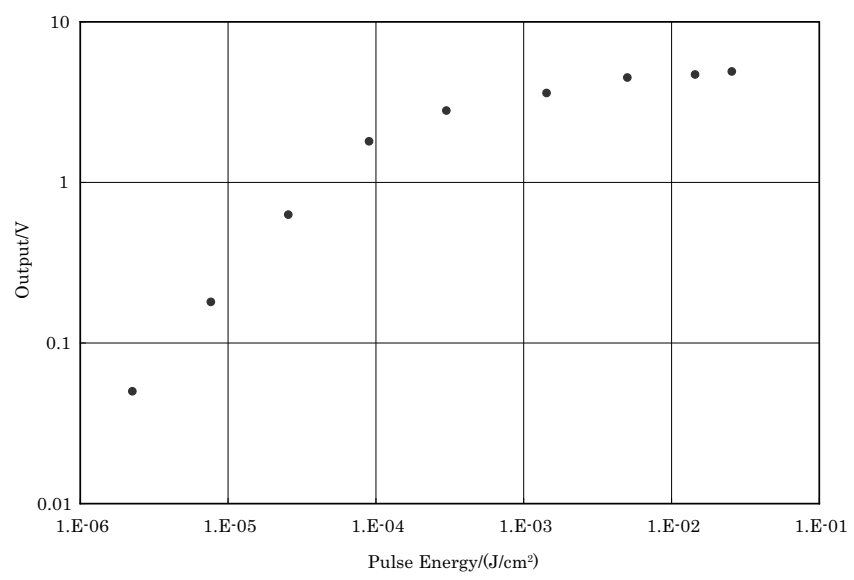

Fig. 3. Dependence on pulse energy of irradiation of ArF excimer laser.

prevent absorption by the sapphire window.

Figure 4 shows the spectral responsivity of the diamond UV photodetector in the wavelength range of 10 to $33 \mathrm{~nm}$. The result shows that the diamond detector is sensitive in the wavelength range of 10 to $33 \mathrm{~nm}$. It was found that the spectral responsivity at $13 \mathrm{~nm}$ wavelength is $6.3 \times 10^{-4} \mathrm{~A} / \mathrm{W}$, and that at $14 \mathrm{~nm}, 6.0 \times 10^{-4} \mathrm{~A} / \mathrm{W}$. It was confirmed that the spectral responsivity was two orders of magnitude higher than that in the case of using dark current. 


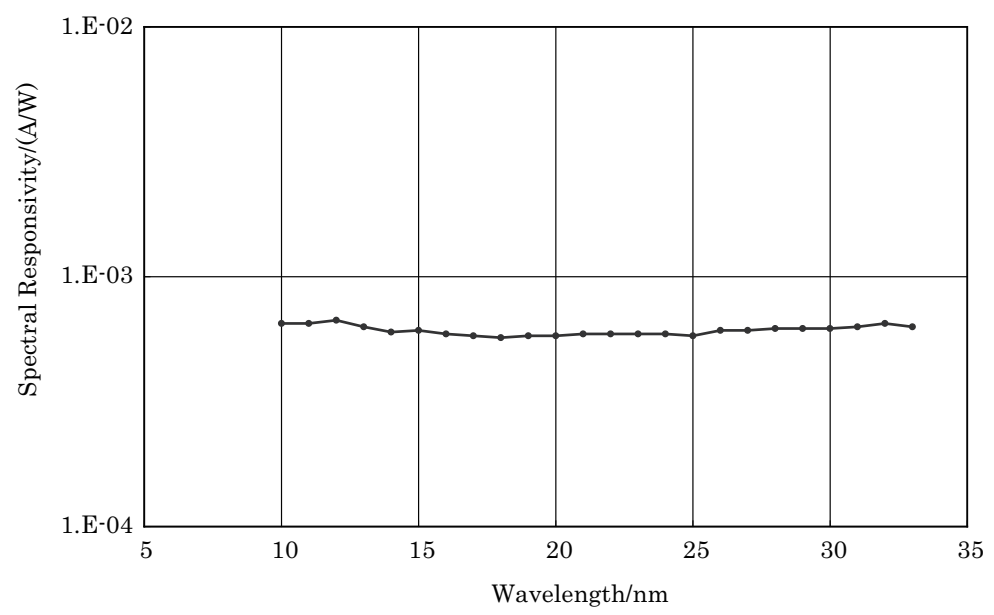

Fig. 4. Spectral responsivity of the diamond UV photodetector in EUV region.

\subsection{Xenon excimer lamp (wavelength: $172 \mathrm{~nm}$ )}

The stability of the spectral responsivity of the diamond UV photodetector upon the irradiation of a xenon excimer lamp (EXH880-1, Iwasaki Electric Co., Ltd.) was evaluated. The measurement was conducted using a commercial product having the readout unit as the UV irradiance meter (EVUV-200, Iwasaki Electric Co., Ltd.), in which a diamond UV photodetector was installed on the light-receiving section.

Figure 5 shows the output of the UV irradiance meter. The irradiance of the detector was measured to be approximately $37 \mathrm{~mW} / \mathrm{cm}^{2}$ and calibrated using the NIST-traceable silicon photodiode. The output signal of the detector was measured every $20 \mathrm{~h}$, but the detector was exposed to radiation from the lamp throughout the test. It was clearly observed that the output signal of the detector was stable even after $700 \mathrm{~h}$.

\subsection{Low-pressure mercury lamp (wavelength: $185 \mathrm{~nm}$ )}

Figure 6 shows the emission spectrum of a typical low-pressure mercury lamp. The low-pressure mercury lamp has an emission peak at a wavelength of $254 \mathrm{~nm}$. The emission intensity at $185 \mathrm{~nm}$ is approximately $30 \%$ of that at $254 \mathrm{~nm}$.

To verify that the diamond UV photodetector is only sensitive to light with a wavelength of $185 \mathrm{~nm}$ emitted from a low-pressure mercury lamp, two types of glass with different transmission limits were inserted between the light source and the diamond UV photodetector, and the output from the photodetector was measured.

Figure 7 shows the transmittance of the two types of glass used as filters in this study. Glass A (ozoneless quartz glass) transmits light of $254 \mathrm{~nm}$ wavelength but blocks that of $185 \mathrm{~nm}$ wavelength, whereas glass B (fluorine-doped synthetic quartz glass) transmits light with wavelengths of both 254 and $185 \mathrm{~nm}$. For measurement, we used 


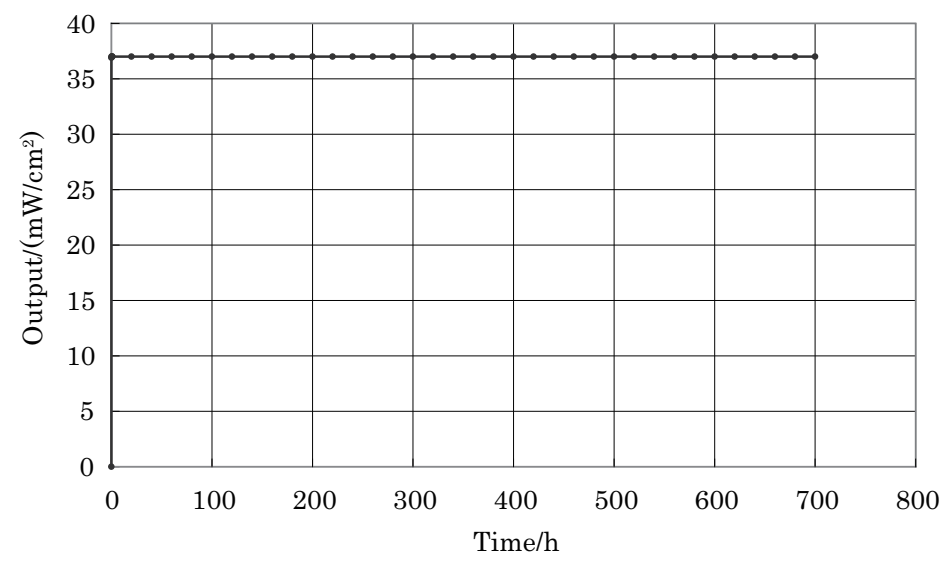

Fig. 5. Stability of spectral responsivity upon continuous radiation of xenon excimer lamp.

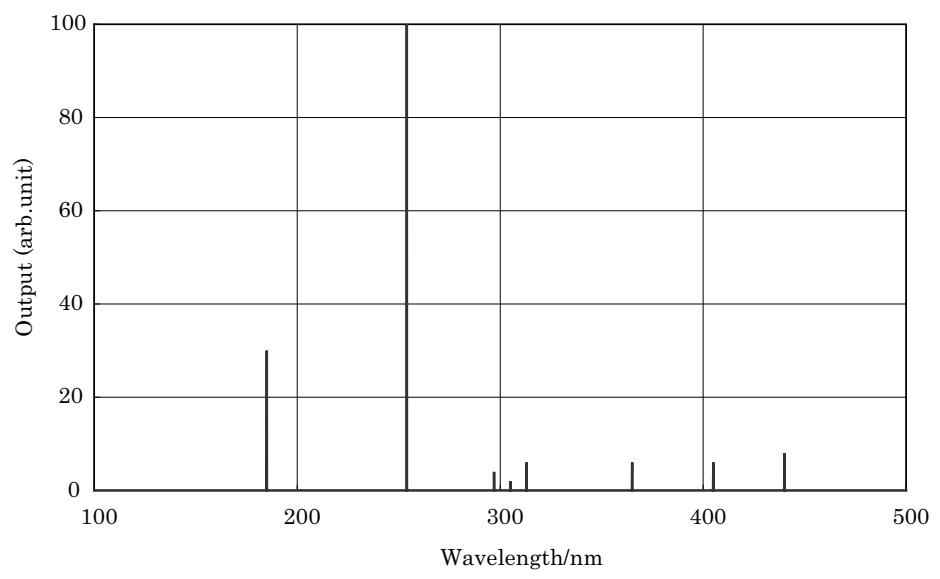

Fig. 6. Typical emission spectrum of low-pressure mercury lamp.

a UV irradiance meter (EVUV-200, Iwasaki Electric Co., Ltd.) and a low-pressure mercury lamp (QGL110U-31, Iwasaki Electric Co., Ltd) as the light source. Figure 8 shows the output of the UV irradiance meter (relative irradiance) when light was irradiated from the low-pressure mercury lamp through the two glass filters. Assuming the relative irradiance without the use of glass (direct irradiation from the low-pressure mercury lamp) as the reference value, the relative irradiance with the use of glass B was approximately $90 \%$ of the reference value, which was equivalent to the transmittance of glass B for the light of $185 \mathrm{~nm}$ wavelength. However, the relative irradiance was 0 when glass A was used. From these results, it was demonstrated that the diamond UV photodetector can detect only light with a wavelength of $185 \mathrm{~nm}$ emitted from a lowpressure mercury lamp. 


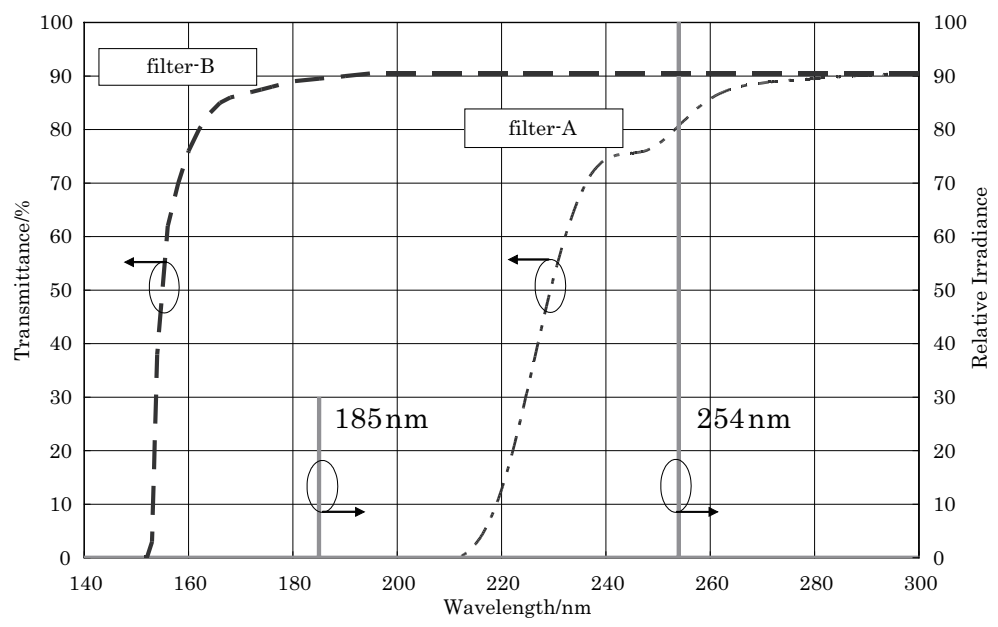

Fig. 7. Transmittance of glass filters used in this experiment.

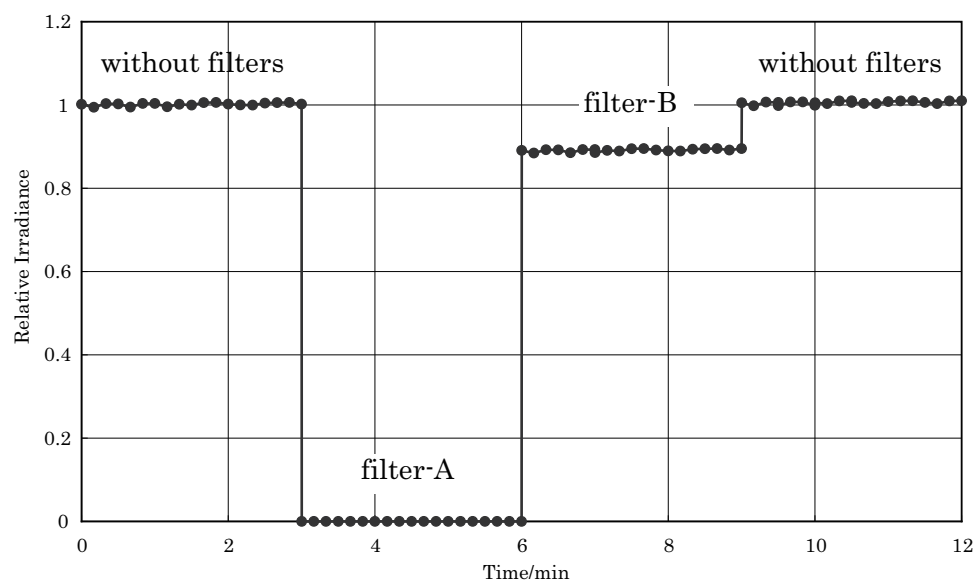

Fig. 8. Measurement of output when glass filters A and B are used.

\section{Conclusions}

The characteristic evaluation of the diamond UV photodetector with a highly oriented diamond film was carried out. For the measurement using an ArF excimer laser, it was confirmed that the diamond UV photodetector can detect a laser with a pulse width of 5 ns and that good linearity between the pulse energy and the output can be obtained in the pulse energy range of $1-100 \mu \mathrm{J} / \mathrm{cm}^{2}$. For the measurement using synchrotron radiation, the spectral responsivity of the diamond UV photodetector was found to be high in 
the wavelength range of $10-33 \mathrm{~nm}$, and it was confirmed that the spectral responsivity was two orders of magnitude higher than that in the case of using dark current. The long-term stability of the spectral responsivity of the diamond UV photodetector was confirmed for the measurement using a xenon excimer lamp, whereas the diamond UV photodetector is only sensitive to light with a wavelength of $185 \mathrm{~nm}$ among lights of different wavelengths irradiated from a low-pressure mercury lamp.

UV light sources have been applied in various industrial fields depending on the wavelength. Diamond UV photodetectors are expected to be applied in photodetectors for the continuous measurement of UV and VUV light sources, the range of applications of which will further expand in the future. The results of the characteristic evaluation indicate that the diamond UV photodetector can be used as a photodetector of light sources for exposure apparatus. Light sources for exposure apparatus have markedly advanced in recent years and their development will continue in the future. The authors expect that the diamond UV photodetector will be applied as a photodetector of light sources for exposure apparatus and will play an important role in the development of next-generation exposure apparatus.

\section{Acknowledgments}

We thank Dr. T. Saito of the National Institute of Advanced Industrial Science and Technology for measuring the spectral responsivity in the EUV spectral range and useful discussion.

\section{References}

1 V. Bakshi (ed.): EUV Sources for Lithography (The Society of Photo-Optical Instrumentation Engineers, Bellingham, 2006).

2 K. Uchida, H. Ishihara, K. Nippashi, M. Matsuoka and K. Hayashi: J. Light Vis. Eng. 28 (2004) 97.

3 K. Hayashi, T. Tachibana, N. Kawakami, Y. Yokota, K. Kobashi, H. Ishihara, K. Uchida, K. Nippashi and M. Matsuoka: Diamond Relat. Mater. 15 (2006) 792.

4 T. Saito and K. Hayashi: Appl. Phys. Lett. 86 (2005) 122.

5 T. Tachibana, K. Hayashi, N. Kawakami, Y. Yokota and K. Kobashi: Appl. Phys. Lett. 68 (1996) 1491.

6 K. Hayashi, T. Tachibana, N. Kawakami, Y. Yokota and K. Kobashi: Diamond Relat. Mater. 10 (2001) 1794.

7 K. Hayashi, T. Tachibana, N. Kawakami, Y. Yokota and K. Kobashi: J. Appl. Phys. 81 (1997) 744.

8 T. Tomimasu, T. Noguchi, S. Sugiyama, T. Yamazaki, T. Mikado and M. Chiwaki: IEEE Trans. Nucl. Sci. NS-30 (1983) 3133.

9 T. Saito and H. Onuki: J. Opt. 24 (1993) 23.

10 T. Saito and H. Onuki: Metrologia 32 (1996) 525.

11 T. Saito, K. Hayashi, H. Ishihara and I. Saito: Metrologia 43 (2006) S51-S55. 